\title{
La contratación pública de obras: situación actual y puntos de mejora
}

\section{Public procurement: current situation and points of improvement}

\author{
J. L. Fuentes-Bargues $^{(*)(* *)}$, C. González-Gaya ${ }^{(* * *)}$, Ma C. González-Cruz $^{(*)}$
}

\section{RESUMEN}

La construcción pública ha sido, es y será uno de los pilares fundamentales del sector construcción español, si bien la actual situación de crisis económica y la reducción de la inversión pública en nuevas infraestructuras ha generado un descenso de actividad hasta niveles similares a los años noventa.

El retorno a índices de actividad más elevados no solo se conseguirá con la recuperación de la economía del país, sino que será necesario realizar ciertas mejoras en los procesos de licitación con objeto de conseguir una mayor funcionalidad y flexibilidad en la contratación pública de obras.

Palabras clave: Contratación pública; construcción; criterios de adjudicación; España; gestión de construcción.

\section{ABSTRACT}

Public construction has been, is and will be one of the fundamental pillars of the Spanish construction sector, while the current economic depression and the reduction of public investment in new infrastructure has led to a decrease of activity as the 9o's.

The return to higher activity rates will be achieved not only with the economic recovery of the country but would be necessary to make some improvements in the bidding process in order to achieve greater functionality and flexibility in the public procurement of works.

Keywords: Public procurement; construction; award criteria; Spain; construction management.

\author{
(*) Universidad Politécnica de Valencia, (España). \\ (**) Universidad de Valencia. Burjassot, Valencia (España). \\ (***) ETSII, UNED. Madrid (España). \\ Persona de contacto/Corresponding author: jofuebar@dpi.upv.es (J. L. Fuentes-Bargues)
}

Cómo citar este artículo/Citation: Fuentes-Bargues, J. L., González-Gaya, C., González-Cruz, Ma C. (2015). La contratación pública de obras: situación actual y puntos de mejora. Informes de la Construcción, 67(537): eo58, doi: http://dx.doi.org/10.3989/ic.12.130.

Licencia / License: Salvo indicación contraria, todos los contenidos de la edición electrónica de Informes de la Construcción se distribuyen bajo una licencia de uso y distribución Creative Commons Reconocimiento no Comercial 3.o. España (cc-by-nc). 


\section{INTRODUCCIÓN}

La construcción en España ha supuesto durante las últimas dos décadas aproximadamente el $10 \%$ del PIB, siendo el principal motor de la economía española. La actual situación económica mundial, junto a las peculiaridades de la economía española y en especial del sector construcción ha generado un desplome de la actividad, tanto en el ámbito privado como en el ámbito público.

Si bien este desplome inicialmente se produjo en el subsector Edificación y en el ámbito privado con el denominado pinchazo de la burbuja inmobiliaria, la situación lejos de mejorar ha generado políticas de ajuste económico y reducción del déficit de los gobiernos nacionales y autonómicos que han reducido el nivel de actividad del sector construcción pública a valores similares a los años noventa.

La situación actual de la contratación pública y la perspectiva futura de crecimiento debe generar que todos los actores intervinientes en el proceso inicien una reflexión sobre aquellos puntos débiles y poco operativos de los procedimientos, con objeto de fijar las bases para una paulatina recuperación.

El objetivo del presente artículo es identificar y describir los principales problemas de la licitación pública española a través del estudio de la normativa, informes de la juntas consultivas de contratación y del análisis de una muestra de licitaciones de obras distribuidas por toda la geografía española, que abarca poderes adjudicadores locales, provinciales, autonómicos y nacionales. Una vez realizado el análisis se identifican puntos de mejora o líneas de actuación para solucionar los problemas detectados. Estos puntos conllevan cambios en la manera de proceder de los órganos de contratación y el desarrollo de futuras líneas de investigación académicas y profesionales.

\section{DATOS GLOBALES DEL SECTOR}

La clasificación más extendida del sector construcción pública es la que subdivide el sector en Ingeniería civil y en Edificación. Las obras contempladas en Ingeniería Civil son aquellas obras diseñadas y/o dirigidas por ingenieros y las obras contempladas en Edificación son aquellas obras diseñadas y/o dirigidas por arquitectos (1).

La relación entre la contratación pública de obras de los subsectores Edificación/Ingeniería Civil ha sido históricamente en torno al 25/75 (2) pasando en los últimos años a una relación más próxima al 30/70. Se destaca el año 2010 con un porcentaje 37/63 debido a la mayor inversión de las entidades locales en obras de Edificación como consecuencia de la dotación económica procedente del gobierno nacional a través del Fondo para la Inversión Local y del Fondo Estatal para el Empleo y Sostenibilidad Local, y de planes similares de gobiernos autonómicos como el Plan de Inversión Productiva de la Generalitat Valenciana.

En el año 2006 la licitación pública alcanzó su máximo histórico con 44.205.305.000 euros (2), manteniendo valores inferiores pero próximos en los años sucesivos (2007 con 37.399.432.00o euros, 2008 con 38.495.264.00o euros y 2009 con 35.354.070.000 euros), pero la necesidad de limitación del endeudamiento del Estado ha frenado la inversión púbica, pasando a 21.960.506.00o euros licitados en el año 2010, a 11.781.358.000 euros licitados en el año 2011 y a 5.931.005.00o euros en el año 2012, lo que supone una reducción del 88,2\% respecto del año 2009 (Figura 1).

La situación económica actual ha generado que los gobiernos europeos y en especial el español hayan tenido que limitar el endeudamiento del Estado y reducir drásticamente la cuantía de la inversión pública en nuevas infraestructuras, con va-

\section{LICITACIÓN OFICIAL ADMINISTRACIONES PÚBLICAS. EDIFICACIÓN E INGENIERÍA CIVIL}

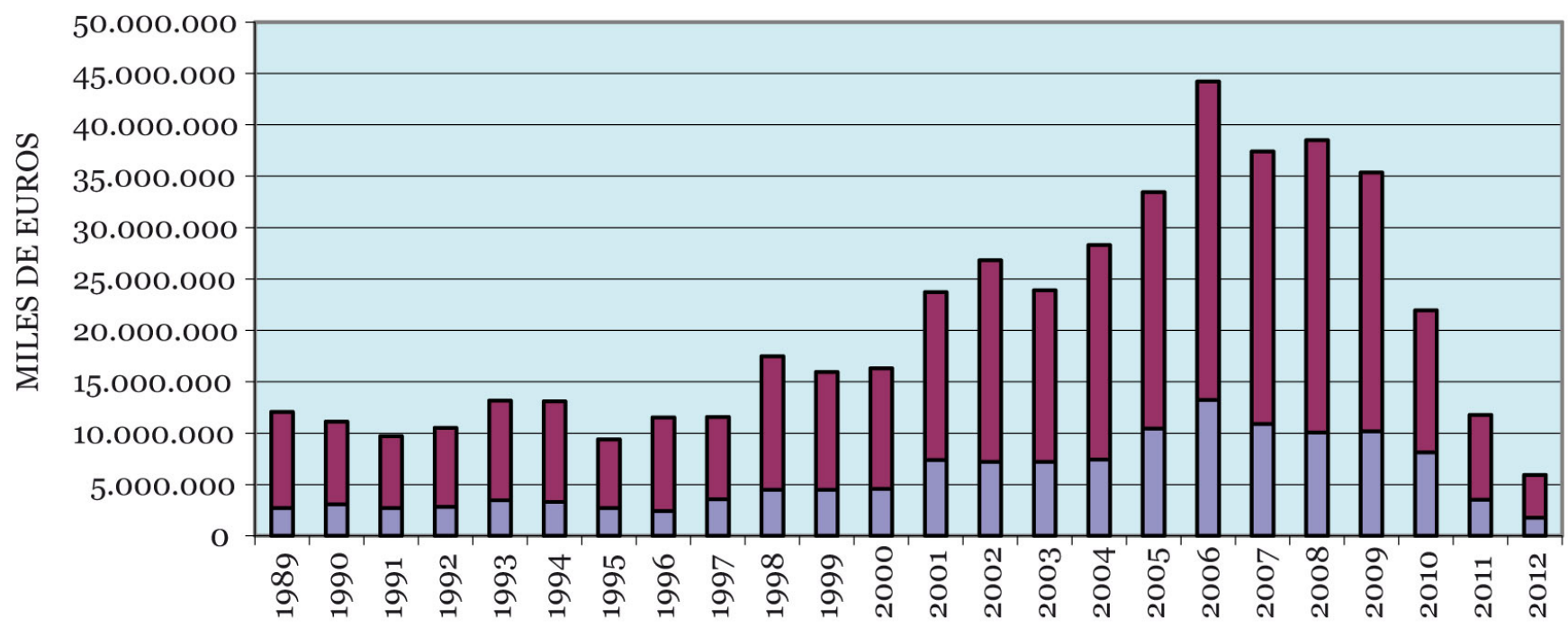

AÑOS

\section{口EDIFICACIÓN口OBRA CIVIL}

Figura 1. Licitación Oficial Administraciones Públicas. Edificación e Ingeniería Civil. Datos: Ministerio de Fomento. 
lores de inversión similares a los años noventa. A pesar de todo, la referencia de la licitación pública dentro del sector construcción español es un hecho.

En cuanto a las tipologías de obras según los dos subsectores se ha de destacar el descenso de la Edificación Residencial dentro del subsector Edificación, que ocupaba tradicionalmente entre un 25-30\%, pasando a un 18,58\% en el año 2010 y a un 22,24\% en el año 2011, fiel reflejo de la reducción de las licitaciones de Viviendas Protegidas debido al gran stock de viviendas existentes en el mercado y a la dificultad de acceso a financiación por parte del usuario final.

En el subsector Ingeniería Civil se ha de destacar la importante inversión que paulatinamente se ha realizado, en especial desde la Administración Central, en infraestructuras ferroviarias, pasando de 540.484.00o euros en el año 1989 a 9.498.892.00o euros en el año 2009 (2), o lo que es lo mismo pasando de que las infraestructuras ferroviarias supusieran el 5,77\% del total de la licitación de la obra de ingeniería civil en el año 1989 a un $37,74 \%$ en el año 2009. El retroceso a nivel económico en la inversión en los últimos tres ejercicios en infraestructura ferroviaria es común a toda la inversión pública, pero porcentualmente sigue manteniendo la cuota respecto al global de la licitación en ingeniería civil, un 38,96\% en el año 2011, lo que pone de manifiesto la apuesta de la administración central por los trazados ferroviarios de alta velocidad.

\section{METODOLOGÍA}

El estudio sobre el estado actual de la construcción pública española se ha abordado por un lado mediante el estudio de la normativa vigente y de los informes de las juntas consultivas de contratación, tanto nacional como autonómicas, y por el otro lado mediante la elaboración y análisis de una muestra de cien licitaciones de obras de construcción públicas, que reflejan diferentes características del sector: los subsectores Obra Civil y Edificación; ámbitos locales, provinciales, autonómicos y nacionales; varios criterios de adjudicación, distribución a lo largo del territorio nacional, etc.

Los documentos obtenidos y analizados para cada uno de los procedimientos de la muestra han sido: los pliegos de cláusulas administrativas y técnicas, el anuncio de licitación y el proyecto de ejecución, al menos el documento presupuesto y el anejo de justificación de costes indirectos.

La muestra de estudio presenta un $53 \%$ de obras del subsector edificación y un $47 \%$ de obras del subsector obra civil. Según el ámbito geográfico, un 16\% de las obras son a nivel nacional, un $37 \%$ a nivel autonómico, un $5 \%$ a nivel provincial y un $42 \%$ a nivel local. Respecto a la cuantía económica de los proyectos estudiados, el $23 \%$ de las obras tienen un presupuesto de licitación ( $\sin$ IVA) entre 200.000 y 1.000.000 de euros, un $44 \%$ entre 1.000.0000 y 5.000.000 de euros, un $22 \%$ entre 5.000.000 de euros y 10.000.0000 de euros y un $11 \%$ superior a 10.000 .000 de euros.

\section{ANÁlISIS DE LA SITUACIÓN ACTUAL}

\subsection{El acceso a la documentación}

La normativa de contratación pública tiene como uno de sus principios básicos la transparencia y acceso público a la información. Para ello la Directiva 2004/18/CE (3), y su transpo- sición al derecho español mediante la Ley 30/2007 (4), creó el perfil del contratante como un punto de información en internet donde cada órgano de contratación debería recoger la información relativa a las licitaciones que lleva a cabo: anuncios previos, pliegos de cláusulas administrativas y técnicas, adjudicaciones de contratos, etc., pero no regula el contenido y alcance de la información.

Esto suscita que algunas administraciones (las menos) pongan a disposición del licitador toda la información (planos, memoria, presupuestos, estudios previos, etc.), mientras que otras administraciones publican la información mínima para la comprensión de la licitación, remitiendo el resto de documentación técnica (proyectos, estudios previos, etc.) a copisterías o similares, para que mediante el pago de un canon se adquiera la documentación.

\subsection{Procedimientos de adjudicación}

La normativa actual (5) contempla varios procedimientos de adjudicación en la tramitación de los contratos públicos: Abierto, donde cualquier empresario que cumpla con los requisitos puede participar; Restringido, donde los empresarios participantes son seleccionados por la administración y no se negocian los términos del contrato; Negociado, donde los empresarios participantes son seleccionados por la administración y se negocian los términos del contrato; y finalmente el Diálogo Competitivo, procedimiento creado por la norma española con objeto de que el sector privado participe en el proceso de desarrollo de las alternativas y soluciones de las diferentes infraestructuras promovidas por el sector público.

La utilización de los procedimientos negociado o restringido viene limitada por las cuantías económicas de los proyectos. Estos sistemas, junto con la adjudicación directa a través de contratos menores, permiten una mayor flexibilidad de la contratación pública, ya que la complejidad y los plazos de los procedimientos son menores, y permite una relación contractual entre las empresas y la administración más semejante a la contratación del sector privado.

Tal y como se refleja en la Figura 2, en la muestra de estudio el procedimiento abierto es el más utilizado con un $94 \%$, representando los procedimientos negociado, restringido y negociado con publicidad solo un $6 \%$, mientras que no aparece el diálogo competitivo.

\section{LICITACIONES SEGÚN TIPO PROCEDIMIENTO}

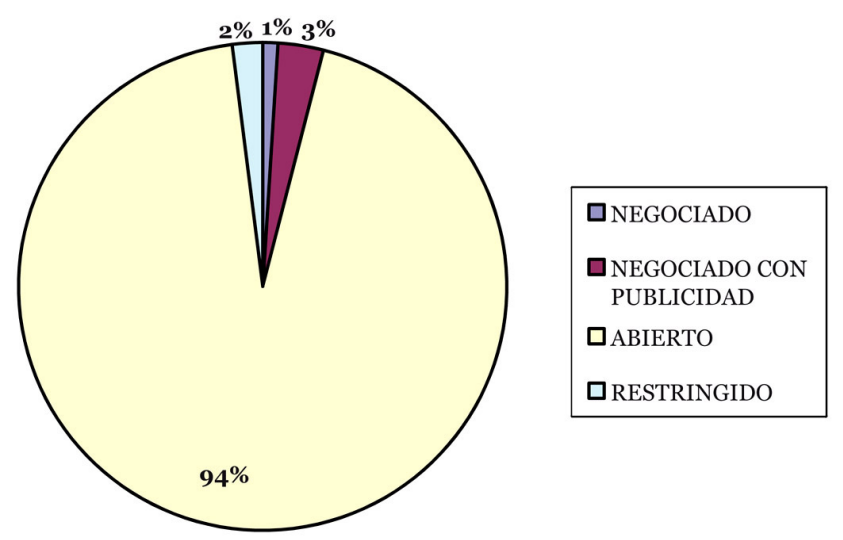

Figura 2. Licitaciones según Tipo Procedimiento. Datos: Muestra de Estudio. 
Si el análisis de la muestra lo realizamos en función de la tipología de obra, en obra civil el $96 \%$ se tramitan como procedimiento abierto y el $4 \%$ como negociado con publicidad, mientras que en edificación el $92 \%$ se tramita como procedimiento abierto, el $4 \%$ como restringido, y los procedimientos negociado y negociado con publicidad tienen un peso del $2 \%$ respectivamente.

\subsection{Formas de Adjudicación}

La actual legislación contempla la adjudicación de las licitaciones bien a la oferta económicamente más ventajosa, atendiendo a un único criterio de adjudicación que necesariamente será el precio, lo que tradicionalmente se ha denominado Subasta o bien atendiendo a varios criterios de adjudicación, como la calidad, el precio, el valor técnico, etc., lo que tradicionalmente se ha denominado Concurso.

La subasta o adjudicación a la oferta económica más baja presenta una simplicidad de procedimiento y de mecanismo para adjudicar el contrato que ha hecho que sea el procedimiento que mejor se ciñe a los principios de transparencia y objetividad que deben regir la contratación pública, pero el tamaño y la complejidad de las obras actuales ha hecho necesario contemplar otros aspectos en la fase de contratación, por lo que su uso se destina a obras de cuantías no muy elevadas y de complejidad baja.

En la muestra de proyectos estudiada el $93 \%$ de los proyectos son concursos frente a un $7 \%$ de subastas. Si se analiza según la tipología de obra, en obra civil el $89 \%$ fueron concursos y el $11 \%$ subastas, mientras que en edificación el $96 \%$ fueron concursos y el $4 \%$ subastas. Estos datos muestran una concordancia con la evolución porcentual de la licitación pública de obras por forma de contratación (Figura 3). Se observa el descenso paulatino de la subasta hasta mantener prácticamente desde hace diez años un $10 \%$ del total de licitaciones públicas de construcción, así como el aumento del número de expedientes tramitados como Negociados desde la aparición de la Ley de Contratos del Sector Público.

La forma de adjudicación más utilizada en la contratación pública española actualmente es el Concurso, donde se valoran di- versos criterios divididos en dos grupos. El primer grupo comprende aquellos criterios que se valoran mediante un juicio de valor y el segundo a aquellos criterios que se valoran mediante fórmulas objetivas. Estos últimos deben suponer más del 50\% del peso de la licitación, sino se debe recurrir a una comisión de expertos ajena al órgano de contratación para evaluar los criterios subjetivos. Normalmente el precio suele ser el único criterio evaluado mediante fórmulas y en raras ocasiones aparece el plazo de ejecución o la mano de obra adscrita al proyecto.

En la Figura 4 se muestra el peso del criterio precio en la muestra de proyectos evaluados en el estudio, donde en un $40 \%$ de la muestra el peso del precio se encuentra entre 51-60 puntos y en un $74 \%$ presenta un peso superior a 50 puntos.

Hay investigaciones discordantes en este sentido que abogan por la utilización de otros parámetros objetivos y una reducción del peso del criterio precio (6) (7), con objeto de conseguir otros beneficios para la administración como procedimientos de ejecución más sostenibles, plazos de ejecución más rápidos o mejoras en el proyecto original.

\subsection{El Precio}

La determinación del precio de las obras es quizás una de las cuestiones más peliagudas de la situación actual. La administración desea realizar más actuaciones o actuaciones más completas que la dotación económica que dispone y se tiende a intentar encajar más proyectos o proyectos más complejos en presupuestos insuficientes, lo que origina problemas durante la ejecución de las obras.

Los licitadores, y más con la situación actual de reducción del número de inversiones, mantienen políticas que se podrían resumir como "coge el contrato y corre» (8) o «firma el contrato y reclama» (9), que se basan en conseguir de cualquier modo (con ofertas desorbitadas o mejoras muy sustanciales para la administración) el contrato y trasladar los problemas económicos a la ejecución de la obra con cuantiosas reclamaciones sobre mediciones erróneas, precios contradictorios, proyectos modificados, etc. (10) (11), que en primer lugar suponen un retraso en la ejecución de las obras y en ocasiones ponen en duda la viabilidad de la obra.

\section{LICITACIÓN CONSTRUCCIÓN POR FORMA CONTRATACIÓN. PORCENTUADOS}

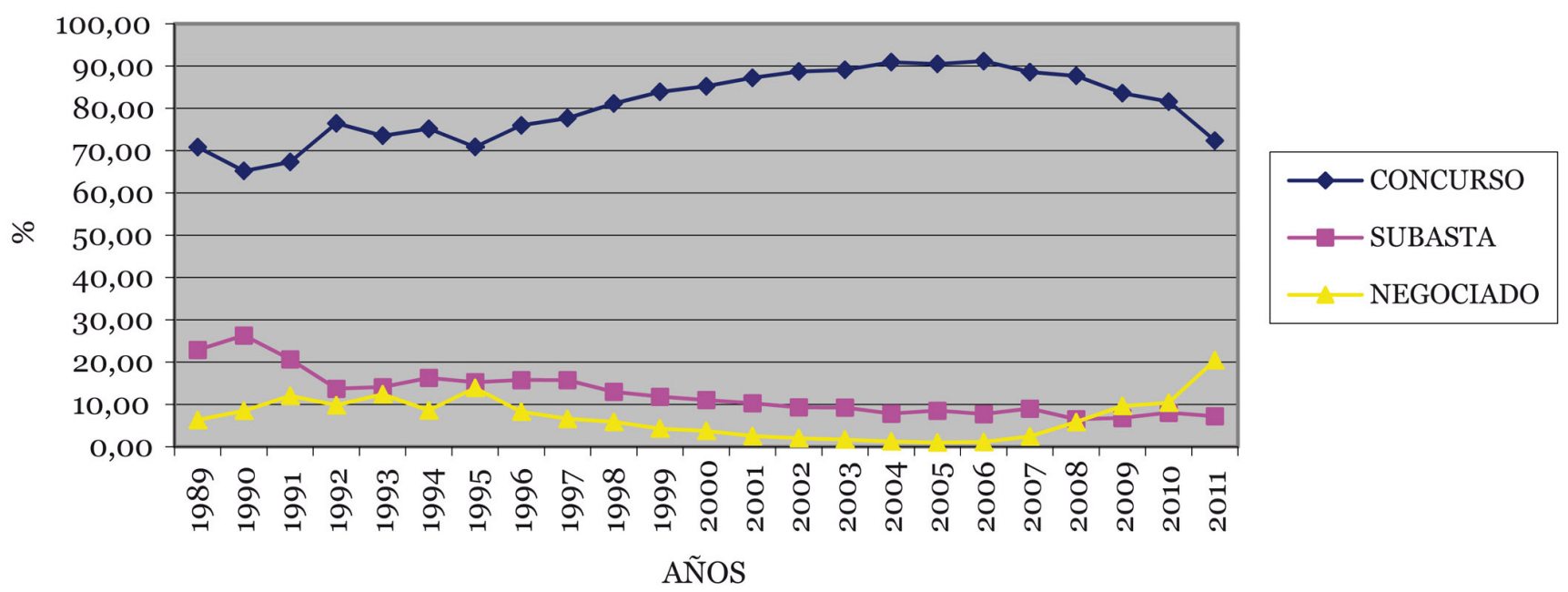

Figura 3. Licitación Construcción por Forma de Contratación. Porcentuados. Datos: Ministerio de Fomento. 


\section{PESO CRITERIO ECONÓMICO RESPECTO TOTAL LICITACIÓN}

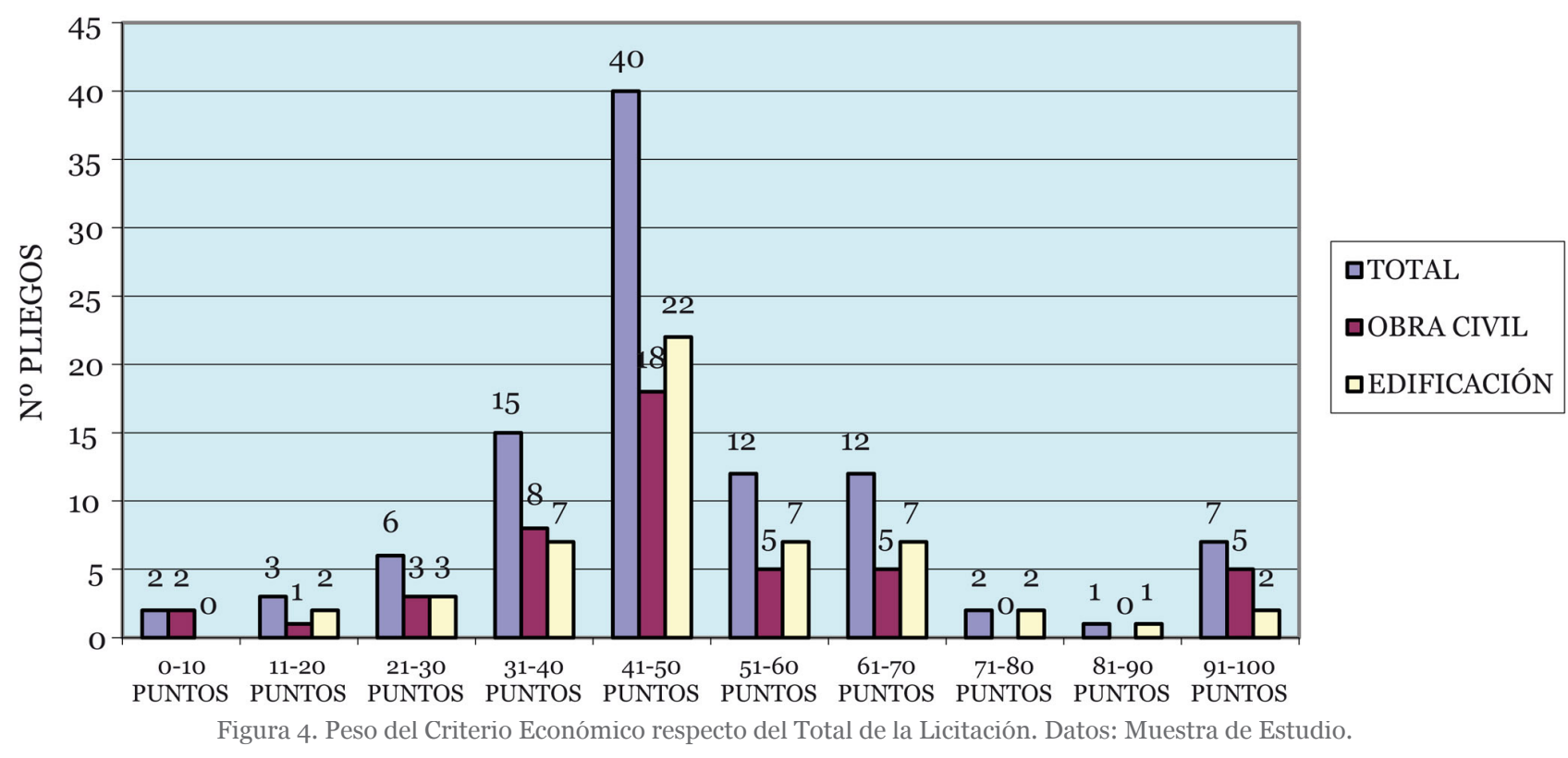

Esta problemática no se ha generado con la actual situación del mercado, sino que con la crisis del sector y la reducción de inversiones se ha acentuado. De hecho en el preámbulo del Real Decreto de Juan Bravo de Murillo (12), normativa precursora del actual sistema de contratación español, se citaba: «La Administración al celebrar contratos no debe proponerse una sórdida ganancia, abusando de las pasiones de los particulares, sino averiguar el precio real de las cosas, y pagar por ello lo que sea justo...».

\subsection{Las Ofertas de Acompañamiento}

El fenómeno de oferta de acompañamiento se puede producir de diferentes modos pero todos con el mismo objetivo, intentar conseguir una posición ventajosa en la puntuación económica de una licitación en base a acuerdos previos con otras empresas competidoras (13) (14).

Por su simplicidad y frecuencia se citan las siguientes situaciones. En un concurso una empresa segura de sus posibilidades de conseguir el contrato por las características de la obra o por su tecnología, solicita a otra empresa con capacidad para contratar (y que hubiera descartado su participación o simplemente su participación fuera meramente testimonial) su participación en la licitación. Para ello suele facilitarle toda la documentación del concurso y en el apartado correspondiente al criterio precio le indica la baja o proposición económica a realizar. Esta proposición económica suele ser baja pero ligeramente superior a la de la empresa interesada, de manera que prácticamente asegura la no incursión en oferta desproporcionada o temeraria de la empresa interesada. Por otra parte respecto al resto de documentación técnica, tanto valorada por fórmulas o mediante un juicio de valor, al ser similar o de inferior calidad le otorgará a la empresa acompañante una menor puntuación. A la empresa interesada, el hecho de obtener una puntuación más elevada en el criterio precio con respecto de otros licitadores y casi asegurando su no incursión en temeridad, puede ser argumento suficiente para contrarrestar posibles proposiciones técnicas de mayor calidad de otros licitadores.
En las subastas sucede de un modo similar pero únicamente con el criterio económico, es decir la empresa interesada en la licitación solicita a otra empresa con la capacidad de contratar la participación en la licitación. Para ello le indica la baja o proposición económica a realizar, siendo esta baja elevada pero ligeramente inferior a la de la empresa interesada, de manera que prácticamente asegura la no incursión en baja desproporcionada o temeraria de la empresa interesada y una posición muy ventajosa, salvo sorpresa de una oferta muy baja de otro licitador.

Estas situaciones no son fáciles de detectar cuando el único de criterio de adjudicación es el precio (subasta), pero sí en cambio cuando existen varios criterios de adjudicación (concurso), ya que parte o casi toda la documentación técnica, relativa a los criterios evaluables mediante juicio de valor, es similar y cuando un técnico o equipo técnico del órgano de contratación revisa la documentación puede comprobar la similitud de las licitaciones en muchos aspectos.

La posición o la decisión de tomar medidas ante estas situaciones es más compleja, ya que por un lado se tienen que dar los suficientes argumentos para excluir de la licitación a ambas propuestas y por otro lado siempre está el beneficio intrínseco que obtiene el órgano de contratación al adjudicar la obra a una proposición económica baja (o en muchos casos a la más baja), con el consiguiente ahorro económico.

\subsection{Empresas dependientes o pertenecientes al mismo grupo empresarial}

En la actualidad, la organización empresarial del sector construcción dibuja un panorama en el que varias empresas independientes funcionalmente entre sí, puedan pertenecer a un mismo grupo empresarial o a un grupo de inversión que controle la mayor o una gran parte del accionariado. Estas empresas, en virtud de los principios normativos de libre acceso y concurrencia a los contratos públicos, pueden presentarse simultáneamente a las licitaciones disponiendo de las mismas oportunidades que cualquier otro empresario con 
la capacidad de contratar, clasificación y solvencia técnica y económica necesaria.

Si se aplica la lógica y la ética en contratación parece evidente que las proposiciones de estas empresas, que aunque en teoría independientes forman parte de un grupo empresarial, se deberían vigilar de manera especial, para evitar el fenómeno de las ofertas de acompañamiento o simplemente evitar que se disponga de dos oportunidades (aspecto claramente contrario a la normativa), y obtener de este modo ventajas respecto del resto de licitadores.

\subsection{Puntuación Máxima Limitada a un porcentaje del Precio de Licitación}

Cuando el criterio económico es el único criterio de adjudicación la oferta económicamente más ventajosa debe ser la baja máxima que no incurra en supuestos de temeridad o desproporcionalidad, pero en los concursos el precio no deja de ser un criterio de decisión más.

El criterio económico o precio debe aparecer como criterio de adjudicación, aunque sea con un reducido peso en el cómputo global de todos los criterios, ya que no se entiende una licitación sin el factor precio (15).

La manera de puntuar el criterio económico en un concurso puede realizarse según diferentes fórmulas o ponderaciones definidas previamente en los pliegos de cláusulas administrativas particulares. Estas fórmulas deben, según la normativa e informes de las juntas consultivas de contratación administrativa, valorar con mayor puntuación a la oferta económica más ventajosa (siempre que no se encuentre en supuestos de desproporcionalidad o anormalidad) y al resto valorarlas proporcionalmente (16) (17) (18) (19).

A pesar de todo de estas reglas claras se siguen utilizando fórmulas de valoración del criterio económico que priman con la máxima puntuación a las ofertas más similares a la oferta media de las proposiciones y al resto se les penaliza conforme se separan de la oferta media, aspecto claramente no aceptado por las autoridades comunitarias (20).

Otras fórmulas de puntuación del criterio económico, también muy utilizadas por muchos órganos de contratación son aquellas que otorgan la máxima puntuación a aquellos licitadores que realizan una baja máxima de un porcentaje respecto del precio de licitación, otorgándoles puntuaciones proporcionales al resto de licitadores que hayan realizado una baja inferior.

La situación que se genera con este tipo de fórmulas económicas tiene sus ventajas para la administración ya que limita la reducción económica en la obra a un máximo (21) que permite, en condiciones normales, al licitador defender la obra ajustando sus costes fijos y gastos generales, sin necesidad de una continua disputa en búsqueda de precios contradictorios, aumento de mediciones y llegado el caso, a proyectos modificados.

Sin embargo, esta baja máxima limitada a un porcentaje sobre el precio de licitación también tiene sus inconvenientes. Todos los licitadores, o la mayor parte de ellos, presentan una oferta económica ajustada a la máxima con objeto de conseguir la totalidad de los puntos del criterio económico y deja la decisión de la adjudicación de la obra principalmente a los criterios emitidos por un juicio de valor (y al resto de criterios evaluables mediante fórmulas, que a parte del precio no suelen ser muchos, normalmente el plazo de ejecución), con las suspicacias que puede generar a las empresas no adjudicatarias, y realmente entrando en una posible situación de falsa «adjudicación directa» o de «reparto de obras» entre los contratistas habituales o de la zona geográfica.

\subsection{La valoración con las proposiciones económicas versus la valoración con los porcentajes de baja}

Aunque pueda parecer un poco paradójico los resultados de valorar o puntuar las ofertas, o comprobar los supuestos de baja temeraria de los licitadores con las ofertas económicas (en euros) o con el porcentaje de baja conduce a resultados distintos, obviamente no se trata de resultados muy dispares, pero si lo suficiente para que empresas con ofertas muy bajas respecto del precio de licitación se encuentren en supuestos de oferta anormal o desproporcionada, o en cambio se encuentren como la empresa mejor posicionada.

Este hecho no es nuevo y la administración ha dado cuenta de ello (22) (23) (24), indicando que en los supuestos de temeridad contemplados en el artículo 85 del Reglamento General de la Ley de Contratos de las Administraciones Públicas - RGLCAP (25) , las expresiones se deberían referir a las ofertas inferiores o superiores en más de diez unidades porcentuales a la media aritmética de las ofertas presentadas y no a la media aritmética de las bajas de las ofertas presentadas.

Consecuencia de las disparidades o agravios que se pueden originar, sería conveniente establecer en los pliegos de contratación, las fórmulas (y el modo de aplicación) de los supuestos de temeridad escogidos para el criterio económico de la licitación y las fórmulas (y el modo de aplicación) de la puntuación o valoración del criterio económico de la licitación.

\subsection{Criterios de Desproporcionalidad o Temeridad}

Los criterios de desproporcionalidad o temeridad permiten determinar ofertas demasiado arriesgadas con respecto al resto de licitadores. Estas fórmulas son determinadas para cada procedimiento por el órgano de contratación y reflejadas en los pliegos de cláusulas del procedimiento concreto. Las fórmulas más usuales se basan en un porcentaje respecto de un precio de referencia (calculado en función de la media y la desviación típica de las bajas o de los importes de las ofertas) o respecto de un porcentaje respecto de la media de las bajas o de la media de los importes de las ofertas de los licitadores (26).

\section{PUNTOS DE MEJORA}

Se sintetizan a continuación una serie de puntos de mejora del proceso de contratación pública de obras respecto de los aspectos descritos en el análisis de la situación actual.

\subsection{El acceso a la documentación}

La tecnología actual permite el acceso a la información de una manera sencilla y a un coste económico muy reducido para las administraciones, por lo que se debería potenciar 
todavía más el uso de internet en la publicidad y en la tramitación de los expedientes de contratación pública, así como la publicación de los datos de las aperturas económicas de las licitaciones o de los datos de las valoraciones técnicas.

\subsection{Procedimientos de adjudicación}

El porcentaje de utilización de procedimientos restringidos, negociados o menores no es muy elevado en comparación con otros sistemas de contratación, en especial los anglosajones, por lo que se deberían potenciar más su uso e incluso aumentar las cuantías que limitan su utilización (6).

El Diálogo Competitivo puede parecer a priori un procedimiento de adjudicación complejo y largo para el sector construcción, pero dada la actual situación podría permitir para grandes obras de infraestructura obtener mejores soluciones técnicas y nuevas fórmulas de financiación o de explotación.

\subsection{Formas de Adjudicación}

Se debe reducir el peso del criterio precio en pro de otros criterios, evaluables mediante fórmulas (plazo, mano de obra) o evaluables mediante un juicio de valor (proceso técnico, control de calidad, criterios medioambientales, etc.) con objeto de obtener mayores beneficios para la administración y en definitiva para la sociedad en general (obras de mayor calidad y sin necesidad de reparaciones posteriores, procesos constructivos más sostenibles que consuman menos recursos y generen menos residuos).

\subsection{El Precio}

Las administraciones deben asegurarse que el precio de la obra a licitar es acorde a los precios de mercado (5), para ello se deberían comparar los diferentes precios de las unidades de obra con precios de bases de construcción de referencia, tanto nacionales elaboradas por empresas privadas como autonómicas elaboradas por organismos dependientes de las administraciones.

\subsection{Las Ofertas de Acompañamiento}

Los órganos de contratación deben establecer pautas o rúbricas a la hora de la evaluación de la documentación técnica de las presentaciones de las diferentes empresas licitadoras (similitud en descripción procesos, en pautas de control de calidad, en alternativas estudiadas, etc.) para determinar el fenómeno de ofertas de acompañamiento en concursos, así como realizar estudios o registros históricos de las proposiciones económicas de las diferentes empresas ofertantes.

\subsection{Empresas dependientes o pertenecientes al mismo grupo empresarial}

Los órganos de contratación para detectar que empresas dependientes o pertenecientes a un mismo grupo empresarial realicen ofertas de acompañamiento o dispongan de dos oportunidades en una licitación deben incluir en los pliegos de cláusulas administrativas particulares una cláusula que actúe sobre el criterio precio, tanto en las subastas como en los concursos, y se aplicaría en el cálculo o la comprobación de los supuestos de baja temeraria. Para el cálculo de la baja media o de la baja de referencia solo se deberá tener en cuen- ta la oferta más económica de todas las presentadas por las empresas pertenecientes al mismo grupo empresarial, con lo cual desaparece la posibilidad de alterar la baja media o la baja de referencia con ofertas muy similares por parte de empresas pertenecientes al mismo grupo empresarial.

\subsection{Puntuación Máxima Limitada a un porcentaje del Precio de Licitación}

Con objeto de evitar los fenómenos de «adjudicación directa» o de «reparto de obras» la fórmula de puntuación del criterio precio no estará limitada a un porcentaje del precio de licitación, sino que se utilizarán fórmulas que otorguen la máxima puntuación a la oferta más económica y al resto de licitadores puntuaciones proporcionales desde cero puntos (si se le otorga esa puntuación a la oferta más elevada) o desde la distancia de la baja realizada al precio de licitación, hasta la máxima puntuación del criterio económico (27).

\subsection{La valoración con las proposiciones económicas versus la valoración con los porcentajes de baja}

No deben existir criterios de desproporcionalidad o fórmulas de puntuación del criterio económico que trabajen con los porcentajes de baja, cualquiera de las utilizadas debe trabajar con las proposiciones económicas.

\subsection{Criterios de Desproporcionalidad o Temeridad}

La selección del criterio de desproporcionalidad o temeridad es una decisión importante del órgano de contratación y es un aspecto muy ligado a la determinación del precio cierto de la licitación.

En lugar de las fórmulas clásicas basadas en una desviación respecto a la oferta media de los licitadores o respecto a una baja de referencia, se podrían utilizar criterios de temeridad o desproporcionalidad de las ofertas basados en introducir limitaciones en los precios de las unidades de obra del proyecto. Obviamente no a todos los precios de las unidades de obra de un proyecto, porque la complejidad del sistema tanto para la administración como para los licitadores sería muy elevada, sino a los precios de las unidades de obras significativas, definiéndose aquellas como las unidades de obra que superen el 10\% del presupuesto de ejecución material que conforma el precio base de licitación de la obra.

A las unidades de obra que cumplan esta condición, que podrían ser por ejemplo unidades correspondientes a estructura (hormigón armado, forjados unidireccionales, etc.) en edificios residenciales o unidades de aglomerado en licitaciones de carreteras, se les podría considerar temerarias si superan un determinado porcentaje (10-15\%) respecto de la media aritmética del precio calculado a partir de todas las proposiciones presentadas.

Se trata de un proceso mucho más complejo para todos los actores de la contratación pero que obligaría en primer lugar al proyectista a un mayor esfuerzo en la redacción y elaboración del documento presupuesto, en segundo lugar a un revisión más ardua por parte de los servicios técnicos del órgano de contratación y en tercer lugar a un estudio pormenorizado de la obra por parte de los departamentos de estudios de las 
empresa constructoras, y no como sucede en la actualidad que no hay una revisión documental del proyecto ni del documento presupuesto salvo que sea uno de los criterios de ponderación del concurso.

\section{CONCLUSIONES}

La Administración Pública debería lograr una mayor flexibilidad y rapidez en los procesos de contratación. Para ello puede de alguna manera copiar o adaptar ciertos procedimientos propios de la contratación en el ámbito privado. Una de las herramientas sería el aumento de las cuantías económicas de los contratos menores, negociados o restringidos para la contratación de obras sencillas o perfectamente definidas y la utilización del diálogo competitivo en obras complejas, que permitiría poner a disposición de la administración el know-how de las empresas contratistas e incluso buscar alternativas a la difícil financiación de la administración pública.
En los pliegos de cláusulas se deben por un lado definir perfectamente todos los aspectos relacionados con el criterio de adjudicación económico: criterios de desproporcionalidad y las fórmulas de puntuación, debiéndose utilizar fórmulas matemáticas que trabajen con las ofertas económicas y no con los porcentajes de baja. Asimismo, se deben eliminar los criterios de desproporcionalidad basados en un porcentaje respecto del precio de licitación y eliminar también las fórmulas de puntuación del criterio precio que otorguen la máxima puntuación a aquellas ofertas más próximas a la media de las ofertas presentadas o que otorguen la máxima puntuación a una oferta con un porcentaje limitado de reducción del precio de licitación.

Por el otro lado se deben definir con mayor exactitud el contenido de los criterios evaluables mediante un juicio de valor e incluso se deberían generar «rúbricas» por parte de los evaluadores de los órganos de contratación que reflejen los atributos o características que se tendrán en cuenta a la hora de emitir un juicio sobre una documentación técnica.

\section{REFERENCIAS}

(1) Alcaide-Inchausti, A., Rodríguez-Saiz, L. (1982). Análisis económico del sector construcción, p. 186, Madrid: Colegio Universitario de Estudios Financieros, Consejo Superior Bancario, D.L.

(2) Ministerio de Fomento. (2013). Licitación Oficial en Construcción. Series mensuales. www.fomento.gob.es/ $\mathrm{BE} /$ ?nivel=2\&orden $=01000000$.

(3) Unión Europea. (2004, 30 de abril). Directiva 2004/18/CE del Parlamento Europeo y del Consejo, de 31 de marzo de 2004, sobre coordinación de los procedimientos de adjudicación de los contratos públicos de obras, de suministro y de servicios. Diario Oficial de la Unión Europea, L 134, pp.114-240.

(4) Jefatura del Estado. (2007, 31 de octubre). Ley 30/2007, de 30 de Octubre, de Contratos del Sector Público. Boletín Oficial del Estado, n²61, pp. 44336-44436. España.

(5) Ministerio de Economía y Hacienda. (2011, 16 de noviembre). Real Decreto Legislativo 3/2011, de 14 de Noviembre, por el que se aprueba el texto refundido de la Ley de Contratos del Sector Público. Boletín Oficial del Estado, $\mathrm{n}^{\circ}$. 276, pp.117729-117914.

(6) Del Caño-Gochi, A., De la Cruz, M. P., De la Cruz, E. M. (2008). Contratación Pública en la Construcción. Madrid: Cie Inversiones-Editorial Dossat 2000 S.L.

(7) Instituto de Estudios Fiscales. (2004). Informe y Conclusiones de la Comisión de Expertos para el Estudio y Diagnóstico de la Situación de la Contratación Pública. Madrid: Ministerio de Hacienda.

(8) Ruiz-Ojeda, A. (Dir.). (2004). Derecho de la contratación pública y regulada, p. 346. Madrid: Editorial Civitas.

(9) Mohamed-Khaled, A., Khoury-Shafik, S., Hafez-Sherif, M., (2011). Contractor's decision for bid profit reduction within opportunistic bidding behaviour of claims recovery. International Journal of Project Management, 29(1): 93-107, doi: http://dx.doi.org/10.1016/j.ijproman.2009.12.003.

(10) Padrós-Reig, C. (2010). La regulación de la baja temeraria en los procedimientos de contratación. Fundamentación actual e historia normativa. Revista de Administración Pública, (181): 267-300.

(11) Martín-Seco, J.F. (2003). El Precio como criterio de adjudicación en los concursos. Cuenta con el IGAE, (7): 11-26.

(12) España. (1852, 29 de febrero). Real Decreto 27 de Febrero de 1852. Gaceta de Madrid, no 6460, pp. 1-2.

(13) Conti, P.L., Naldi, M. (2008). Detection of anomalous bids in procurement auctions. Decision Support Systems, 46(1): 420-428, doi: http://dx.doi.org/10.1016/j.dss.2008.08.002.

(14) Ballesteros-Pérez, P., González-Cruz, M., Cañabate-Grimal, A., Pellicer, E., (2013). Detecting abnormal and collusive bids in capped tendering. Automation in Construction, (31): 215-229, doi: http://dx.doi.org/10.1016/j.autcon.2012.11.036.

(15) JCCA. (1998). Informe 27/98, de 11 de Noviembre. Ponderación del criterio del precio en concursos. Junta Consultiva de Contratación Administrativa de España.

(16) Junta Consultiva de Contratación Administrativa del Gobierno Balear. (1998). Informe 05/98, de 26 de Junio, Valoración del Precio como Criterio de Adjudicación.

(17) JCCA. (1998). Informe 29/98, de 11 de Noviembre, Diversas cuestiones relativas a los criterios de adjudicación de concursos en los contratos de obras. Junta Consultiva de Contratación Administrativa de España.

(18) Junta Consultiva de Contratación Administrativa de la Comunidad de Madrid. (200o). Informe 1/20oo, de 8 de Febrero, Sobre la ponderación del criterio precio en los concursos.

(19) JCCA. (2011). Informe 04/11, de 28 de Octubre. Consulta sobre baremar las ofertas económicas en un pliego de cláusulas administrativas particulares valorando con mayor puntuación, en cuanto al precio, a ofertas que no se corresponden con la más baja. Junta Consultiva de Contratación Administrativa de España.

(20) Gimeno-Feliu, J.M. (2008). La Adjudicación de los contratos: La oferta económicamente más ventajosa. Monografías de la Revista Aragonesa de Administración Pública, 10: 155-184. 
(21) Fueyo-Bros, M. (2009). Criterios objetivos de valoración versus objetivos de los criterios de adjudicación. El Consultor de los Ayuntamientos y de los Juzgados, (15-16): 2196-2280.

(22) JCCA. (2004). Informe 47/O3, de 2 de Febrero de 2004. Aplicación del artículo 85.4 del Reglamento General de la Ley de Contratos de las Administraciones Públicas en la apreciación de bajas desproporcionados o temerarias. Junta Consultiva de Contratación Administrativa de España.

(23) Comisión Consultiva de Contratación Administrativa de la Junta de Andalucía. (2003). Recomendación 8/2002, de 4 de Febrero de 2003, sobre interpretación de los artículos 85 y 86 del Reglamento general de la Ley de Contratos de las Administraciones Públicas aprobado por el Real Decreto 1098/2001, de 12 de Octubre.

(24) Junta Regional de Contratación Administrativa de la Región de Murcia. (2002). Consulta sobre la interpretación del artículo 85 del Real Decreto 1098/2001, de 12 de Octubre, por el que se aprueba el Reglamento General de la Ley de Contratos de las Administraciones Públicas. Informe 02/2002, de 16 de Julio.

(25) Ministerio de Hacienda. (2011, 26 de octubre). Real Decreto 1098/2001, de 12 de Octubre, por el que se aprueba el Reglamento general de la Ley de Contratos de las Administraciones Públicas. Boletín Oficial del Estado, nº. 257, pp. 39252-39371.

(26) Fuentes-Bargues, J.L., González-Gaya, C. (2013). Determination of Disproportionate Tenders in Public Procurement. Journal of Investment and Management. 2(1): 1-9, doi: http://dx.doi.org/10.11648/j.jim.20130201.11.

(27) Fuentes-Bargues, J.L., González-Gaya, C. (2013). Analysis of the Scoring Formula of Economic Criteria in Public Procurement. Journal of Economic Behavior and Organization. 1(1): 1-12, doi: http://dx.doi.org/10.11648/j.ijebo.20130101.11. 